\title{
On the One Class of Hyperbolic Systems
}

Vsevolod E. ADLER and Alexey B. SHABAT

L.D. Landau Institute for Theoretical Physics, 1 A prosp. ak. Semenova, 142432 Chernogolovka, Russia

E-mail: adler@itp.ac.ru,shabat@itp.ac.ru

Received October 27, 2006; Published online December 27, 2006

Original article is available at http://www.emis.de/journals/SIGMA/2006/Paper093/

Abstract. The classification problem is solved for some type of nonlinear lattices. These lattices are closely related to the lattices of Ruijsenaars-Toda type and define the Bäcklund auto-transformations for the class of two-component hyperbolic systems.

Key words: hyperbolic systems; Bäcklund transformations; Ruijsenaars-Toda lattice; discrete Toda lattice

2000 Mathematics Subject Classification: 35L75; 35Q55; 37K10; 37K35

This paper is dedicated to the memory of Vadim Kuznetsov

\section{Introduction}

In this paper we solve the problem of classification of the consistent pairs of the lattices

$$
\begin{aligned}
& u_{x}=F\left(u_{1}, u, v\right), \quad v_{x}=G\left(u, v, v_{-1}\right), \\
& u_{y}=P\left(u_{-1}, u, v\right), \quad v_{y}=Q\left(u, v, v_{1}\right) .
\end{aligned}
$$

Here $u=u(n, x, y), v=v(n, x, y), n \in \mathbb{Z}$ and subscripts denote both the partial derivatives with respect to $x, y$ and the shift with respect to $n$. The nondegeneracy conditions are assumed

$$
F_{v} F_{u_{1}} G_{u} G_{v_{-1}} P_{v} P_{u_{-1}} Q_{u} Q_{v_{1}} \neq 0 .
$$

Due to the condition $F_{v} \neq 0$, the first equation of the lattice (1) can be solved with respect to the variable $v$, then the second equation of the lattice rewrites as a lattice of the Ruijsenaars-Toda type $[1,2,3,4]$

$$
u_{x x}=A\left(u_{1, x}, u_{x}, u_{-1, x}, u_{1}, u, u_{-1}\right)
$$

while its symmetry takes the form

$$
u_{y}=B\left(u_{x}, u_{1}, u, u_{-1}\right) .
$$

Clearly, the roles of $x$ and $y$ may be interchanged and equation (4) may be replaced by a lattice of the form

$$
u_{y y}=C\left(u_{1, y}, u_{y}, u_{-1, y}, u_{1}, u, u_{-1}\right) .
$$

The classification problem for the lattices (4) was solved, in a different setting, in our paper [5], see also $[6,7,8,9]$. The approach based on the relation to the lattices (1) allows to reproduce

${ }^{\star}$ This paper is a contribution to the Vadim Kuznetsov Memorial Issue "Integrable Systems and Related Topics". The full collection is available at http://www.emis.de/journals/SIGMA/kuznetsov.html 
this result in a slightly more general way and is promising for further generalizations. The classification of the integrable lattices of the form (1) was obtained by Yamilov [13]. The main postulate in his work was that, instead of (2), a symmetry of a high enough order exists, and moreover, the lattice was assumed to be Hamiltonian. Our aim here is to obtain the answer under the minimal restrictions. However, in comparison with the result of Yamilov, our list contains only two more pairs. These pairs are not Hamiltonian, and they are reducible in some definite sense. We discuss these curious examples separately in Section 5 .

The interest to the lattices (1) is explained by their close relation to many other integrable models, see e.g. [10, 11, 12]. The higher symmetries of such lattices generate the hierarchies of the evolution systems of the nonlinear Schrödinger type. In this context equation (2) defines the first negative flow of the hierarchy and corresponds to some hyperbolic system, see Section 4.1. Although not all integrable two-component hyperbolic systems can be obtained in this way, this correspondence is a source of important examples. Also, the linear combinations of the flows (1), (2) constitute a class of equations containing Ablowitz-Ladik and Sklyanin lattices $[14,15]$.

In Section 4.3 we demonstrate that the transform to the Ruijsenaars-Toda lattice (4) exhibits a hidden discrete symmetry of the lattice (1). Namely, it turns out that the lattices of the form (4) for the variables $u$ and $v$ coincide (after an appropriate substitution), and this allows to expand the equations onto the square grid and brings to the discrete Toda lattices $[16,17,18]$. It should be noted that conversely, the lattices of the form (1) or (4) can be obtained from the discrete Toda type equations under the continuous limits [19].

Section 7 contains the list of the consistent Hamiltonian pairs (1), (2) and the corresponding lists of the hyperbolic systems and Ruijsenaars-Toda lattices.

Returning to the setting of the problem, notice that it is natural to consider equivalent lattices related by the point substitutions

$$
\tilde{u}=\phi(u), \quad \tilde{v}=\psi(v)
$$

the scalings $\tilde{x}=\alpha x, \tilde{y}=\beta y$ and the renamings

$$
\begin{array}{llll}
u \leftrightarrow v, & n \leftrightarrow-n, & F \leftrightarrow G, & P \leftrightarrow Q, \\
x \leftrightarrow y, & n \leftrightarrow-n, & F \leftrightarrow P, & G \leftrightarrow Q .
\end{array}
$$

We use these transforms in order to bring a lattice to a simpler form. Moreover, the substitution

$$
\tilde{u}=u+\alpha x+\beta y+\gamma n, \quad \tilde{v}=v+\alpha x+\beta y+\gamma n
$$

is often useful. It is clear that it brings, in general, to a nonautonomous lattice. However, if the right hand sides of the lattices contain only the differences $u-v, u_{ \pm 1}-v$ then this transformation preserves the class under consideration. For brevity, we refer to all mentioned transformations as to admissible substitutions. Our main result is the following theorem.

Theorem 1. The consistent lattices (1), (2), such that the nondegeneracy conditions (3) are fulfilled, are brought by the admissible substitutions to one of the Hamiltonian pairs in the list 7.1,

$$
\begin{array}{lll}
u_{x}=a(u, v) \delta_{v} H, & v_{x}=-a(u, v) \delta_{u} H, & H=K\left(u_{n+1}, v_{n}\right)+L\left(u_{n}, v_{n}\right), \\
u_{y}=a(u, v) \delta_{v} R, & v_{x}=-a(u, v) \delta_{u} R, & R=M\left(u_{n}, v_{n+1}\right)+N\left(u_{n}, v_{n}\right)
\end{array}
$$

$\left(\delta_{u} H=\partial_{u} \sum_{n} T^{n}(H)\right.$ denotes the lattice variational derivative), or to one of the pairs

$$
u_{x}=\frac{v+(1+\varepsilon) u+u_{1}}{\varepsilon v-u_{1}}, \quad v_{x}=\frac{v_{-1}+\left(1+\varepsilon^{-1}\right) v+u}{v_{-1}-\varepsilon^{-1} u},
$$




$$
\begin{aligned}
& u_{y}=\frac{v+\left(1+\varepsilon^{-1}\right) u+u_{-1}}{\varepsilon^{-1} v-u_{-1}}, \quad v_{y}=\frac{v_{1}+(1+\varepsilon) v+u}{v_{1}-\varepsilon u}, \\
& u_{x}=f\left(v-u_{1}\right), \quad v_{x}=\varepsilon f\left(v_{-1}-u\right), \\
& u_{y}=p\left(\varepsilon u_{-1}-v\right), \quad v_{y}=p\left(\varepsilon u-v_{1}\right)
\end{aligned}
$$

or to the linear lattices.

\section{Necessary conditions}

In this Section we deduce some consequences from the compatibility condition of the lattices under consideration. Let $T$ denote the shift operator $n \rightarrow n+1$.

Proposition 1. If the lattices (1), (2) commute then a constant $\varepsilon$ and functions $a(u, v)$, $b\left(u, u_{1}\right), c\left(v, v_{1}\right), h\left(u_{1}, v\right), r\left(u, v_{1}\right)$ exist and are unique up to the scaling $(a, b, c, h, r) \rightarrow$ $(k a, k b, k c, h / k, r / k)$, such that

$$
\begin{aligned}
& F_{u_{1}}=a h, \quad F_{v}=b h, \\
& G_{v_{-1}}=-\varepsilon a T^{-1}(h), \quad G_{u}=-T^{-1}(c h), \\
& P_{u_{-1}}=-\varepsilon a T^{-1}(r), \quad P_{v}=-T^{-1}(b r), \\
& Q_{v_{1}}=a r, \quad Q_{u}=c r .
\end{aligned}
$$

Proof. Computing $u_{x y}$ in two ways gives the equation

$$
F_{u_{1}} T(P)+F_{u} P+F_{v} Q=P_{u_{-1}} T^{-1}(F)+P_{u} F+P_{v} G .
$$

Differentiating of this expression with respect to $u_{1}$ and $u_{-1}$ yields $F_{u u_{1}} P_{u_{-1}}=P_{u u_{-1}} F_{u_{1}}$, which implies

$$
F_{u_{1}}=a(u, v) h\left(u_{1}, v\right), \quad P_{u_{-1}}=a(u, v) \hat{r}\left(u_{-1}, v\right) .
$$

Using the symmetry (8) gives analogously

$$
G_{v_{-1}}=\tilde{a}(u, v) \hat{h}\left(u, v_{-1}\right), \quad Q_{v_{1}}=\tilde{a}(u, v) r\left(u, v_{1}\right) .
$$

Let us prove that, moreover, the following relations hold

$$
\begin{aligned}
& T\left(P_{v}\right)=-b r, \quad \tilde{a} F_{v}=a b h, \\
& T^{-1}\left(Q_{u}\right)=-\hat{c} \hat{r}, \quad a G_{u}=\tilde{a} \hat{c} \hat{h}, \\
& T^{-1}\left(F_{v}\right)=-\hat{b} \hat{h}, \quad \tilde{a} P_{v}=a \hat{b} \hat{r}, \\
& T\left(G_{u}\right)=-c h, \quad a Q_{u}=\tilde{a} c r
\end{aligned}
$$

with some functions

$$
b=b\left(u, u_{1}\right), \quad \hat{c}=\hat{c}\left(v_{-1}, v\right), \quad \hat{b}=\hat{b}\left(u_{-1}, u\right), \quad c=c\left(v, v_{1}\right) .
$$

In order to obtain the first line, differentiate (17) with respect to $v_{1}$. This yields $F_{v} Q_{v_{1}}+$ $F_{u_{1}} T\left(P_{v}\right)=0$, or $\tilde{a} F_{v} /(a h)=-T\left(P_{v}\right) / r$. Here the left and right hand sides depend respectively on $u_{1}, u, v$ and $u, u_{1}, v_{1}$, so that their common value is some function $b\left(u, u_{1}\right)$. In order to prove the rest, it is sufficient to use the symmetries (8), (9).

Now, one easily obtains from (18) the relation

$$
\frac{a(u, v)}{\tilde{a}(u, v)} \cdot \frac{b\left(u, u_{1}\right)}{T\left(\hat{b}\left(u_{-1}, u\right)\right)}=\frac{T(a(u, v))}{T(\tilde{a}(u, v))} \cdot \frac{c\left(v, v_{1}\right)}{T\left(\hat{c}\left(v_{-1}, v\right)\right)} .
$$


This implies $a / \tilde{a}=\psi(v) / \phi(u)$. One may assume $a=\tilde{a}$ without loss of generality, taking into account the substitution

$$
a \rightarrow a \psi, \quad \tilde{a} \rightarrow \tilde{a} \phi, \quad h \rightarrow h / \psi, \quad \hat{r} \rightarrow \hat{r} / \psi, \quad \hat{h} \rightarrow \hat{h} / \phi, \quad r \rightarrow r / \phi .
$$

Then $b=\varepsilon T(\hat{b}), c=\varepsilon T(\hat{c})$ with some constant $\varepsilon$ and functions $h, \hat{h}$ and $r, \hat{r}$ are related by equations

$$
\hat{h}=-\varepsilon T^{-1}(h), \quad \hat{r}=-\varepsilon T^{-1}(r) .
$$

Taking all together proves the required equations.

The system of equations (13)-(16) is overdetermined and further information about the form of the lattices will be obtained mainly by analysis of the compatibility conditions of this system. However, this cannot solve the problem completely, since the functions $F, P$ and $G, Q$ are found from this system at least up to the addition of arbitrary functions on $u$ and $v$ respectively. Therefore, after some point we will need additional information. It turns out that use of the following proposition allows to determine the right hand sides of the lattices up to few arbitrary constants. The final answer is obtained then by the intermediate check of the consistency.

Proposition 2. If the lattices (1), (2) commute then the following equations hold

$$
\begin{aligned}
& h_{v} Q+h_{u_{1}} T(P)=-h\left(T\left(P_{u}\right)+Q_{v}+k_{1}\right), \quad a_{u} P+a_{v} Q=a\left(P_{u}+Q_{v}+k_{1}\right), \\
& r_{u} F+r_{v_{1}} T(G)=-r\left(F_{u}+T\left(G_{v}\right)+k_{2}\right), \quad a_{u} F+a_{v} G=a\left(F_{u}+G_{v}+k_{2}\right),
\end{aligned}
$$

where $k_{1}, k_{2}$ are some constants and the functions $a, r, h$ are defined in the Proposition 1.

Proof. Equations (22), (23) are equivalent to the conservation laws

$$
\begin{array}{lr}
D_{y}\left(\log F_{u_{1}}\right)=(1-T)\left(P_{u}\right), & D_{y}\left(\log G_{v_{-1}}\right)=\left(1-T^{-1}\right)\left(Q_{v}\right), \\
D_{x}\left(\log P_{u_{-1}}\right)=\left(1-T^{-1}\right)\left(F_{u}\right), & D_{y}\left(\log Q_{v_{1}}\right)=(1-T)\left(G_{v}\right),
\end{array}
$$

where the left hand sides are replaced in accordance to the formulae (13)-(16). In its turn, the first of these conservation laws is obtained by differentiating of (17) with respect to $u_{1}$, and in order to obtain the other ones it is sufficient to apply the symmetries (8), (9).

Remark 1. We see, comparing the equations (11) and (13), (14) that for the Hamiltonian lattices $h=H_{u_{1} v}$ and $\varepsilon=1$. In the general case parameter $\varepsilon$ remains undetermined almost till the end of calculations. This makes necessary to consider several additional branches with $\varepsilon \neq 1$, leading to essential complication of the analysis (see Propositions 4, 5 below). Only in two of these cases the solution is not empty. Moreover, it is not difficult to prove that in the Hamiltonian case the second column of the equations (22), (23) turns into identities and the constants $k_{1}, k_{2}$ vanish.

In what follows we use the substitutions (7). They act on the functions under consideration in accordance to the rules

$$
\begin{aligned}
& \tilde{F}=\phi^{\prime}(u) F, \quad \tilde{G}=\psi^{\prime}(v) G, \quad \tilde{P}=\phi^{\prime}(u) P, \quad \tilde{Q}=\psi^{\prime}(v) Q, \\
& \tilde{h}=h /\left(\psi^{\prime}(v) \phi^{\prime}\left(u_{1}\right)\right), \quad \tilde{r}=r /\left(\phi^{\prime}(u) \psi^{\prime}\left(v_{1}\right)\right), \\
& \tilde{a}=\phi^{\prime}(u) \psi^{\prime}(v) a, \quad \tilde{b}=\phi^{\prime}(u) \phi^{\prime}\left(u_{1}\right) b, \quad \tilde{c}=\psi^{\prime}(v) \psi^{\prime}\left(v_{1}\right) c .
\end{aligned}
$$




\section{Proof of the classification theorem}

We will see soon that it is convenient to divide all lattices into two classes, depending on whether $a$ is of the form $a(u, v)=a_{1}(u) a_{2}(v)$ or not. We start from the case when this factorization does not hold. The form of the lattices from this subclass (which contain the larger part of the list) is defined more exactly in the following proposition.

Proposition 3. If the lattice (1) satisfies the equations (13), (14) at $a a_{u v} \neq a_{u} a_{v}$ then it can be brought, by a substitution (7), to one of the following types:

$$
\begin{aligned}
& \left\{\begin{array}{l}
u_{x}=a u_{1}+f_{1}(u) v+f(u), \\
v_{x}=-\varepsilon a v_{-1}+g_{1}(v) u+g(v),
\end{array} \quad a=\sum_{i, j=0}^{1} a_{i j} u^{i} v^{j},\right. \\
& \left\{\begin{array}{l}
u_{x}=\frac{a}{v-u_{1}}-\frac{a_{v}}{2}+f(u), \\
v_{x}=\frac{\varepsilon a}{v_{-1}-u}+\frac{\varepsilon a_{u}}{2}+g(v),
\end{array} \quad a=\sum_{i, j=0}^{2} a_{i j} u^{i} v^{j} .\right.
\end{aligned}
$$

Proof. Cross-differentiating of the relations (13) yields

$$
a_{v} h+a h_{v}=b_{u_{1}} h+b h_{u_{1}} .
$$

Dividing by $h$ and applying the operator $\partial_{v} \partial_{u_{1}}-(\log h)_{v u_{1}}$ yields

$$
a N_{u_{1}}=b M_{v}, \quad N=\frac{h_{v v}}{h}-\frac{3 h_{v}^{2}}{2 h^{2}}, \quad M=\frac{h_{u_{1} u_{1}}}{h}-\frac{3 h_{u_{1}}^{2}}{2 h^{2}} .
$$

Differentiating with respect to $u$ gives $a_{u} N_{u_{1}}=b_{u} M_{v}$. Since $a a_{u v} \neq a_{u} a_{v}$, hence $a_{u} b \neq a b_{u}$ and therefore $M_{v}=N_{u_{1}}=0$, that is $M=M\left(u_{1}\right), N=N(v)$. It is easy to obtain the rule of transformation of these functions under the substitution $(7)$ :

$$
M=\left(\phi^{\prime}\right)^{2} \tilde{M}+\frac{\phi^{\prime \prime \prime}}{\phi^{\prime}}-\frac{3\left(\phi^{\prime \prime}\right)^{2}}{2\left(\phi^{\prime}\right)^{2}}, \quad N=\left(\psi^{\prime}\right)^{2} \tilde{N}+\frac{\psi^{\prime \prime \prime}}{\psi^{\prime}}-\frac{3\left(\psi^{\prime \prime}\right)^{2}}{2\left(\psi^{\prime}\right)^{2}}
$$

We set $M=N=0$ by an appropriate choice of $\phi, \psi$. Denote $h=H^{-2}$, then these equalities and equation (28) take the form

$$
H_{v v}=0, \quad H_{u_{1} u_{1}}=0, \quad a_{v} H-2 a H_{v}=b_{u_{1}} H-2 b H_{u_{1}} .
$$

Notice that we may still apply the Möbius transformations of $v$ and $u_{1}$. This allows to bring $H$ to one of the forms $H=1$ or $H=v-u_{1}$ (depending on whether the original polynomial is reducible or not).

If $h=1$ then the system (13) takes the form $F_{u_{1}}=a(u, v), F_{v}=b\left(u, u_{1}\right)$, whence

$$
F=\alpha(u) v u_{1}+\beta(u) v+\gamma(u) u_{1}+\delta(u)
$$

Analogously,

$$
G=\varkappa(v) v_{-1} u+\lambda(v) v_{-1}+\mu(v) u+\nu(v),
$$

moreover $-\varepsilon a=G_{v_{-1}}=-\varepsilon F_{u_{1}}$, and equations (26) follow.

If $h=\left(v-u_{1}\right)^{-2}$ then one easily finds from equations (29) that $a=a_{2} v^{2}+a_{1} v+a_{0}$, $b=-a_{2} u_{1}^{2}-a_{1} u_{1}-a_{0}$, where the coefficients depend on $u$. Solving the system (13) yields

$$
F=\frac{\alpha(u) v u_{1}+\beta(u) v+\gamma(u) u_{1}+\delta(u)}{v-u_{1}}
$$


where $\alpha=a_{2}, \beta+\gamma=a_{1}, \delta=a_{0}$. Analogously,

$$
G=\frac{\varkappa(v) v_{-1} u+\lambda(v) v_{-1}+\mu(v) u+\nu(v)}{v_{-1}-u}
$$

and one proves that $a$ is a biquadratic polynomial by comparing the formulae

$$
a=\alpha(u) v^{2}+(\beta(u)+\gamma(u)) v+\delta(u), \quad \varepsilon a=\varkappa(v) u^{2}+(\lambda(v)+\mu(v)) u+\nu(v) .
$$

The lattice takes the form (27) with $2 f=\beta-\gamma, 2 g=\lambda-\mu$.

The similar statement is valid for the lattice (2) as well, due to the symmetry (9). However, the substitutions (7) for both lattices may be different, so that the second one should be written for some another variables $U=U(u), V=V(v)$ :

$$
\begin{aligned}
& \left\{\begin{array}{l}
U_{y}=-\varepsilon A U_{-1}+p_{1}(U) V+p(U), \\
V_{y}=A V_{1}+q_{1}(V) U+q(V),
\end{array} \quad A=\sum_{i, j=0}^{1} A_{i j} U^{i} V^{j},\right. \\
& \left\{\begin{array}{l}
U_{y}=\frac{\varepsilon A}{U_{-1}-V}+\frac{\varepsilon A_{V}}{2}+p(U), \\
V_{y}=\frac{A}{U-V_{1}}-\frac{A_{U}}{2}+q(V),
\end{array}\right.
\end{aligned}
$$

The lattices (26), (27) and (30), (31) combine into three variants of the pairs, up to the symmetry (9). In each case some additional information about the right hand sides can be obtained by comparing the formulae for the functions $a, b, c$ corresponding to both lattices. The following lemma is useful as well. Here we denote

$$
\tilde{F}\left(U_{1}, U, V\right)=U^{\prime} F\left(u_{1}, u, v\right), \quad \tilde{G}\left(U, V, V_{-1}\right)=V^{\prime} G\left(u, v, v_{-1}\right)
$$

for the right hand sides of the lattice (1) rewritten in the variables $U, V$.

Lemma 1. If the lattice (1) is of the form (26) then its symmetry (2) satisfies the conditions $P_{u u}=Q_{v v}=0$. If the lattice (1) is of the form (27) then $P_{u u u}=Q_{v v v}=0$ and, moreover, $P$ and $Q$ coincide as the functions on their arguments, that is $P\left(u, v, v_{1}\right)=Q\left(u, v, v_{1}\right)$.

Analogously, if the lattice (2) is of the form (30) then $\tilde{F}_{U U}=\tilde{G}_{V V}=0$, and if the lattice (2) is of the form (31) then $\tilde{F}_{U U U}=\tilde{G}_{V V V}=0$ and $\tilde{F}\left(U_{1}, U, V\right)=\tilde{G}\left(U_{1}, U, V\right)$.

Proof. Let us use the first of the relations (22). If $h=1$ then the equalities $P_{u u}=Q_{v v}=0$ follow immediately. If $h=\left(v-u_{1}\right)^{-2}$ then differentiating with respect to $u_{1}$ gives

$$
Q=T(P)+\left(v-u_{1}\right) T\left(P_{u}\right)+\frac{1}{2}\left(v-u_{1}\right)^{2} T\left(P_{u u}\right) .
$$

Differentiating once more yields $P_{\text {uuu }}=0$. Moreover, the last equation is nothing but the Taylor expansion in the second argument for the function $P\left(u, v, v_{1}\right)$. The equations for $F, G$ follow in virtue of the symmetry (9).

Proposition 4. The consistent lattices with the condition $a a_{u v} \neq a_{u} a_{v}$ are brought by the admissible substitutions to one of the pairs $\left(X_{1}, Y_{1}\right)-\left(X_{4}, Y_{4}\right)$ or $\left(X_{7}, Y_{7}\right)$.

Proof. 1) Let the lattices be of the form (27), (31). Then one can set $h=\left(v-u_{1}\right)^{-2}$ and $r=\left(U-V_{1}\right)^{-2} U^{\prime} V_{1}^{\prime}$, after scaling of $y$. The comparison of the functions $a, b, c$ gives

$$
a=a(u, v)=\frac{A(U, V)}{U^{\prime} V^{\prime}}, \quad-b=a\left(u, u_{1}\right)=\varepsilon \frac{A\left(U_{1}, U\right)}{U_{1}^{\prime} U^{\prime}}, \quad-c=\varepsilon a\left(v, v_{1}\right)=\frac{A\left(V_{1}, V\right)}{V_{1}^{\prime} V^{\prime}},
$$


where

$$
a(u, v)=\sum_{i, j=0}^{2} a_{i j} u^{i} v^{j}, \quad A(U, V)=\sum_{i, j=0}^{2} A_{i j} U^{i} V^{j} .
$$

The differentiation of the first equation yields

$$
\begin{aligned}
& \left(a_{u} U^{\prime}+a U^{\prime \prime}\right) V^{\prime}=A_{U} U^{\prime}, \quad\left(a_{u u} U^{\prime}+2 a_{u} U^{\prime \prime}+a U^{\prime \prime \prime}\right) V^{\prime}=A_{U U}\left(U^{\prime}\right)^{2}+A_{U} U^{\prime \prime}, \\
& \left(3 a_{u u} U^{\prime \prime}+3 a_{u} U^{\prime \prime \prime}+a U^{I V}\right) V^{\prime}=3 A_{U U} U^{\prime} U^{\prime \prime}+A_{U} U^{\prime \prime \prime}
\end{aligned}
$$

and elimination of $A_{U}, A_{U U}$ brings to the relation

$$
\left(2 U^{\prime \prime \prime} U^{\prime}-3\left(U^{\prime \prime}\right)^{2}\right) U^{\prime} a_{u}=\left(4 U^{\prime \prime \prime} U^{\prime \prime} U^{\prime}-U^{I V}\left(U^{\prime}\right)^{2}-3\left(U^{\prime \prime}\right)^{3}\right) a .
$$

Since $a a_{u v} \neq a_{u} a_{v}$ this implies $2 U^{\prime \prime \prime} U^{\prime}-3\left(U^{\prime \prime}\right)^{2}=0$, that is $U$ is a linear-fractional function. The same is true for $V$ as well.

Since the lattice (31) is form invariant under the substitution $\tilde{U}=M(U), \tilde{V}=M(V)$ where $M$ is an arbitrary Möbius transform, hence one may set $U=u$ without loss of generality. Then one may apply the Möbius transformations to the variables: $\tilde{u}=M(u), \tilde{v}=M(v)$. Under this transformation the function $V$ is changed in accordance to the formula $\tilde{V}=M V M^{-1}$, so that it can be brought to one of the forms $V(v)=\delta v$ or $V(v)=v+2 \delta$. The system (32) becomes equivalent to the equation $a(u, v)=\varepsilon A(v, u)$ which defines $A$ and the relation

$$
\varepsilon a(u, v) V^{\prime}(v)=a(V(v), u)
$$

which means that function $a$ possesses some generalized symmetry property. All biquadratic polynomials which satisfy this identity can be found directly. They are listed in the Table 1 (up to the inversion $(u, v) \rightarrow(1 / u, 1 / v)$; the restrictions on the parameters are introduced in order to avoid the intersections and to provide the condition $a a_{u v} \neq a_{u} a_{v}$ ). Now we only have to find the coefficients of $f, g, p, q$ which are quadratic polynomials, in accordance to the Lemma 1. This is done by the direct computations which show that the most cases are empty. As a result, we obtain the pairs $\left(X_{1}, Y_{1}\right),\left(X_{7}, Y_{7}\right)$ and also the pairs

$$
\begin{aligned}
& u_{x}=\frac{a}{v-u_{1}}+u-v+\beta, \quad v_{x}=\frac{a}{v_{-1}-u}+u-v+\beta, \\
& u_{y}=\frac{a}{u_{-1}-v-2 \delta}-u+v+\gamma, \quad v_{y}=\frac{a}{u-v_{1}-2 \delta}-u+v+\gamma,
\end{aligned}
$$

where $a=(u-v-\delta)^{2}+\alpha$, and

$$
\begin{aligned}
& u_{x}=\frac{a}{v-u_{1}}-\delta v+\beta u, \quad v_{x}=\frac{a}{v_{-1}-u}+u+(\beta+\alpha) v, \\
& u_{y}=\frac{\delta a}{u_{-1}-\delta v}+\delta v+(\gamma+\alpha) u, \quad v_{y}=\frac{a}{u-\delta v_{1}}-u+\gamma v,
\end{aligned}
$$

where $a=u^{2}+\alpha u v+\delta v^{2}$. However, these lattices can be brought to the particular cases of the lattice $\left(X_{1}, Y_{1}\right)$ by the transformation (10). For the lattices (33) one should apply the substitution

$$
u \rightarrow u+(\beta+\delta) x+(\gamma-\delta) y-\delta(n-1), \quad v \rightarrow v+(\beta+\delta) x+(\gamma-\delta) y-\delta n
$$

and the new variables satisfy the lattices $\left(X_{1}, Y_{1}\right)$ with $a=(u-v)^{2}+\alpha$. For $(34)$, the analogous substitution is conjugated by exponentiation. 
Table 1. Solutions of the equation $\varepsilon a(u, v) V^{\prime}(v)=a(V(v), u)$.

\begin{tabular}{|c|c|c|c|}
\hline$\varepsilon$ & $V(v)$ & $a(u, v)$ & \\
\hline-1 & $v$ & $\begin{array}{c}a_{22} u^{2} v^{2}+a_{12} u v(u+v)+a_{02}\left(u^{2}+v^{2}\right) \\
\quad+a_{11} u v+a_{01}(u+v)+a_{00} \\
(u-v)(\alpha u v+\beta(u+v)+\gamma)\end{array}$ & $\begin{array}{c}\left(X_{1}, Y_{1}\right) \\
-\end{array}$ \\
\hline $\begin{array}{r}1 \\
-1\end{array}$ & $\begin{array}{l}v+2 \delta \\
v+2 \delta\end{array}$ & $\begin{array}{l}(u-v-\delta)^{2}+\alpha, \quad \delta \neq 0 \\
u-v-\delta, \quad \delta \neq 0\end{array}$ & $\begin{array}{l}(33) \\
-\end{array}$ \\
\hline $\begin{array}{r}\sqrt[3]{1} \\
\sqrt{-1} \\
-1 \\
1 \\
-1\end{array}$ & $\begin{array}{l}\varepsilon v \\
-v \\
-v \\
\delta v \\
\delta v \\
\varepsilon^{-2} v\end{array}$ & $\begin{array}{l}\alpha u^{2} v^{2}+\varepsilon u+v, \quad \alpha \neq 0 \\
\alpha u v(u+\varepsilon v)+\beta(\varepsilon u+v), \quad \alpha \beta \neq 0 \\
\alpha u^{2} v^{2}+\beta\left(u^{2}+v^{2}\right)+\gamma, \quad \beta \gamma \neq \alpha^{2} \\
u^{2}+\alpha u v+\delta v^{2}, \quad \delta \neq 0,1 \\
u^{2}-\delta v^{2}, \quad \delta \neq 0,1 \\
\varepsilon u+v, \quad \varepsilon^{2} \neq 1\end{array}$ & $\begin{array}{c}- \\
- \\
- \\
(34) \\
- \\
\left(X_{7}, Y_{7}\right)\end{array}$ \\
\hline
\end{tabular}

2) Let the lattices be of the form (27), (30), $h=\left(v-u_{1}\right)^{-2}, r=U^{\prime} V_{1}^{\prime}$. Then

$$
\begin{aligned}
& a(u, v)=\sum_{i, j=0}^{2} a_{i j} u^{i} v^{j}=\frac{A_{11} U V+A_{10} U+A_{01} V+A_{00}}{U^{\prime} V^{\prime}}, \\
& b\left(u, u_{1}\right)=-a\left(u, u_{1}\right)=\frac{\varepsilon A_{11} U U_{1}+\varepsilon A_{01} U-p_{1}\left(U_{1}\right)}{U^{\prime} U_{1}^{\prime}}, \\
& c\left(v, v_{1}\right)=-\varepsilon a\left(v, v_{1}\right)=\frac{A_{11} V V_{1}+A_{10} V_{1}+q_{1}(V)}{V^{\prime} V_{1}^{\prime}} .
\end{aligned}
$$

As in the previous case, the first equation implies that functions $U, V$ are linear-fractional. The other two equations are equivalent to relations

$$
\begin{aligned}
& \frac{A_{11} V(v)+A_{10}}{V^{\prime}(v)}=-\varepsilon \frac{A_{11} U(v)+A_{01}}{U^{\prime}(v)}, \\
& \frac{p_{1}(U(u))}{U^{\prime}(u)}=\frac{A_{01} V(u)+A_{00}}{V^{\prime}(u)}, \quad \frac{q_{1}(V(v))}{V^{\prime}(v)}=-\varepsilon \frac{A_{10} U(v)+A_{00}}{U^{\prime}(v)} .
\end{aligned}
$$

We assume, without loss of generality, that either $A=U V+1$ or $A=\varepsilon U-V$. Equation (35) implies $U(u)=c V(u)^{-\varepsilon}$ (and, consequently, $\varepsilon= \pm 1$ ) in the first case, or $U(u)=V(u)+c$ in the second one. The appropriate substitutions, the Möbius one in the lattice (27) and the linear one in (30), reduce the problem to the following cases:

$$
\begin{aligned}
& \varepsilon=1, U=u, \quad V=v^{-1}, \quad a=-v(u+\delta v), \quad A=U V+\delta, \quad \delta \neq 0 ; \\
& \varepsilon=-1, \quad U=u, \quad V=v, \quad a=A=u v+1 ; \\
& U=u, \quad V=v, \quad a=A=\varepsilon u-v+\delta .
\end{aligned}
$$

Moreover, the functions $p_{1}, q_{1}$ are defined from the relations (36) and functions $f(u), g(v), p(u)$, $q(V)$ from the equations

$$
f^{\prime \prime}=0, \quad\left(\left(V^{\prime} g\right)^{\prime} / V^{\prime}\right)^{\prime}=0, \quad p^{\prime \prime \prime}=0, \quad\left(q / V^{\prime}\right)_{v v}=0,
$$

in accordance to the Lemma 1. In the first case, after defining the coefficients by the direct computation and the substitutions $u \rightarrow e^{u}, v \rightarrow e^{v}$ and (10) we obtain the lattices $\left(X_{3}, Y_{3}\right)$. The 
second case turns out to be empty and the third one contains, at $\varepsilon=1$, the pair $\left(X_{2}, Y_{2}\right)$, up to the admissible transformations.

3) Let the lattices be of the form (26), (30), $h=1$ and $r=U^{\prime} V_{1}^{\prime}$. The comparison of the expressions for the function $a$ brings to the equation

$$
\left(a_{11} u v+a_{10} u+a_{01} v+a_{00}\right) U^{\prime} V^{\prime}=A_{11} U V+A_{10} U+A_{01} V+A_{00} .
$$

Computing $a a_{u v}-a_{u} a_{v}$ one obtains the relation

$$
\left(a_{11} a_{00}-a_{10} a_{01}\right) U^{\prime} V^{\prime}=A_{11} A_{00}-A_{10} A_{01}
$$

which implies $U^{\prime \prime}=V^{\prime \prime}=0$. Therefore, we may assume, after the linear substitutions, $U=u$, $V=v, A=a$. Lemma 1 says that the right hand sides of both lattices are linear in any variable. Several equations for the coefficients follow from the relations for the functions $b, c$ :

$$
\begin{aligned}
& b\left(u, u_{1}\right)=a_{11} u u_{1}+a_{01} u_{1}+f_{1}(u)=\varepsilon a_{11} u u_{1}+\varepsilon a_{01} u-p_{1}\left(u_{1}\right), \\
& c\left(v, v_{1}\right)=\varepsilon a_{11} v v_{1}+\varepsilon a_{10} v-g_{1}\left(v_{1}\right)=a_{11} v v_{1}+a_{10} v_{1}+q_{1}(v) .
\end{aligned}
$$

After the final check of the compatibility condition we arrive to the pair $\left(X_{4}, Y_{4}\right)$.

In order to finish the classification we have to consider the lattices with the function $a$ of the form $a(u, v)=a_{1}(u) a_{2}(v)$.

Proposition 5. The consistent lattices with the condition $a a_{u v}=a_{u} a_{v}$, are brought by the admissible substitutions to one of the pairs $\left(X_{5}, Y_{5}\right),\left(X_{6}, Y_{6}\right)$ or $\left(X_{8}, Y_{8}\right)$.

Proof. The substitution (7) allows to reduce the problem (temporarily) to the case $a=1$. Then the compatibility conditions for the systems (13)-(16) are

$$
\frac{h_{v}}{h}=b_{u_{1}}+b \frac{h_{u_{1}}}{h}, \quad \varepsilon \frac{h_{u_{1}}}{h}=c_{v}+c \frac{h_{v}}{h}, \quad \varepsilon \frac{r_{v_{1}}}{r}=b_{u}+b \frac{r_{u}}{r}, \quad \frac{r_{u}}{r}=c_{v_{1}}+c \frac{r_{v_{1}}}{r} .
$$

As a corollary, the equations hold

$$
b_{u}(\log h)_{v u_{1}}=0, \quad c_{v_{1}}(\log h)_{v u_{1}}=0, \quad b_{u_{1}}(\log r)_{u v_{1}}=0, \quad c_{v}(\log r)_{u v_{1}}=0 .
$$

We will use also the following consequences of the relations (22), (23):

$$
P_{u}+Q_{v}+k_{1}=0, \quad F_{u}+G_{v}+k_{2}=0
$$

1) At first, assume that both quantities $(\log h)_{v u_{1}}$ and $(\log r)_{u v_{1}}$ do not vanish. Then $b, c$ are constants and $b c=\varepsilon$. A scaling of $u$ and $v$ allows to set $b=-1, c=-\varepsilon$. One obtains, after the integration of the equations (13)-(16) and taking (38) into account, the lattices of the form

$$
\begin{aligned}
& u_{x}=f\left(v-u_{1}\right)+\alpha_{1} u+\alpha, \quad v_{x}=\varepsilon f\left(v_{-1}-u\right)+\beta_{1} v, \\
& u_{y}=p\left(\varepsilon u_{-1}-v\right)+\gamma_{1} u+\gamma, \quad v_{y}=p\left(\varepsilon u-v_{1}\right)+\delta_{1} v .
\end{aligned}
$$

The direct computation proves (notice that $f^{\prime \prime} p^{\prime \prime} \neq 0$ by assumption) that the linear terms are zero and brings to the pair $\left(X_{8}, Y_{8}\right)$.

2) Now let either $(\log h)_{v u_{1}}=0$ or $(\log r)_{u v_{1}}=0$. Taking the symmetry (9) into account, we assume for definiteness that $h=m\left(u_{1}\right) n(v)$. Then the two first equations (37) take the form

$$
\frac{n^{\prime}}{n}=b_{u_{1}}+b \frac{m^{\prime}}{m}, \quad \varepsilon \frac{m^{\prime}}{m}=c_{v}+c \frac{n^{\prime}}{n},
$$


whence

$$
m^{\prime}=\mu m, \quad n^{\prime}=\nu n, \quad b_{u_{1}}=\nu-\mu b, \quad c_{v}=\varepsilon \mu-\nu c .
$$

Here we have to consider several subcases.

2.1) Let $\mu \nu \neq 0$. After scaling, assume $\mu=1, \nu=-1, h=e^{u_{1}-v}$. Then

$$
b=e^{-u_{1}} \bar{m}(u)-1, \quad c=e^{v} \bar{n}\left(v_{1}\right)-\varepsilon,
$$

and two last equations (37) are reduced to relations

$$
\bar{m}^{\prime}(u) r+\bar{m}(u) r_{u}=0, \quad \bar{n}^{\prime}\left(v_{1}\right) r+\bar{n}\left(v_{1}\right) r_{v_{1}}=0, \quad r_{u}+\varepsilon r_{v_{1}}=0 .
$$

If $\bar{m}=\bar{n}=0$ then we obtain the lattice of the form $\left(X_{8}, Y_{8}\right)$. Otherwise,

$$
b=\beta e^{-\delta \varepsilon u-u_{1}}-1, \quad c=\gamma e^{v+\delta v_{1}}-\varepsilon, \quad r=\lambda e^{\delta\left(\varepsilon u-v_{1}\right)},
$$

where at least one of the coefficients $\beta, \gamma$ is not zero. The solution of equations (13), (14) is

$$
F=e^{u_{1}-v}-\beta e^{-\delta \varepsilon u-v}+f(u), \quad G=\varepsilon e^{u-v_{-1}}-\gamma e^{u+\delta v}+g(v) .
$$

The functions $P, Q$ are easily found as well. One easily proves, by use of (38), that $\delta=-1$, $\varepsilon=1, \beta=\gamma$ and the functions $f, g$ are linear. After the direct computation of the constants and the substitution $e^{u} \rightarrow u, e^{-v} \rightarrow v$ (of course, it spoils the gauge $a=1$ ) we come to the pair $\left(X_{5}, Y_{5}\right)$.

2.2) Let $\mu=0, \nu \neq 0$. Taking $\nu=1, h=e^{v}$ we obtain

$$
\begin{aligned}
& b=u_{1}+\bar{m}(u), \quad c=e^{-v} \bar{n}\left(v_{1}\right), \\
& r_{u}=0, \quad \bar{n}^{\prime}\left(v_{1}\right) r+\bar{n}\left(v_{1}\right) r_{v_{1}}=0, \quad \varepsilon r_{v_{1}}=\bar{m}^{\prime}(u) r .
\end{aligned}
$$

From here, $b=u_{1}+\varepsilon \delta u+\beta, c=\gamma e^{-v-\delta v_{1}}, r=\lambda e^{\delta v_{1}}$ and the solution of equations (13), (14) is

$$
F=\left(u_{1}+\delta \varepsilon u+\beta\right) e^{v}+f(u), \quad G=-\varepsilon e^{v_{-1}}-\gamma u e^{-\delta v}+g(v) .
$$

The second equation (38) now reads $\delta \varepsilon e^{v}+f^{\prime}(u)+\gamma \delta u e^{-\delta v}+g^{\prime}(v)+k_{2}=0$. Since $\gamma \neq 0$ in virtue of the nondegeneracy condition (3), hence $\delta=0$. Taking $r=1$ and solving equations (15), (16) one obtains

$$
P=-\varepsilon u_{-1}-(u+\beta) v+p(u), \quad Q=v_{1}+\gamma u e^{-v}+q(v) .
$$

However, then the first equation (38) implies $\gamma=0$. Therefore, this case is empty (as well as the case $\mu \neq 0, \nu=0$, due to the symmetry (8)).

2.3) Finally, let $\mu=\nu=0$. Then $b=b(u), c=c\left(v_{1}\right)$ and one can take $h=1$. Then $F=u_{1}+b(u) v+f(u), G=-\varepsilon v_{-1}-c(v) u-g(v)$ and substitution into (38) yields

$$
b^{\prime}(u) v+f^{\prime}(u)-c^{\prime}(v) u-g^{\prime}(v)+k_{2}=0 .
$$

From here,

$$
b(u)=\alpha u^{2}+2 \beta_{1} u+\beta, \quad c(v)=\alpha v^{2}+2 \gamma_{1} v+\gamma .
$$

Moreover, elimination of $r$ from the equations (37) brings to the relation

$$
\varepsilon b^{\prime \prime}+\left(b^{\prime 2}-b b^{\prime \prime}\right) c=\varepsilon^{2} c^{\prime \prime}+\varepsilon\left(c^{2}-c c^{\prime \prime}\right) b, \quad b=b(u), \quad c=c\left(v_{1}\right) .
$$


Denote $\Delta_{b}=b^{\prime 2}-2 b b^{\prime \prime}=4\left(\beta_{1}^{2}-\alpha \beta\right)$, and analogously for $c$, then this relation takes the form

$$
2 \varepsilon \alpha+\left(\Delta_{b}+2 \alpha b\right) c=2 \varepsilon^{2} \alpha+\varepsilon\left(\Delta_{c}+2 \alpha c\right) b .
$$

If $\alpha \neq 0$ then it follows that $\varepsilon=1$ and $\Delta_{b}=\Delta_{c}=0$, that is $b$ and $c$ are full squares. The linear change of variables brings them to the form $b=u^{2}, c=v_{1}^{2}$. Then one finds from (37) that $r=\left(u v_{1}-1\right)^{-2}$ and solution of the equations (15), (16) is $P=u_{-1} /\left(u_{-1} v-1\right)+p(u)$, $Q=-v_{1} /\left(u v_{1}-1\right)+q(v)$. Equations (38) say that functions $f, g, p, q$ are linear and after the direct computation of the constants we obtain the pair $\left(X_{6}, Y_{6}\right)$.

If $\alpha=0$ then $\beta_{1} \gamma_{1}=0, \beta_{1}^{2} \gamma=\varepsilon \gamma_{1}^{2} \beta$. Since functions $b, c$ do not vanish in virtue of (3), hence $\beta_{1}=\gamma_{1}=0$, that is the lattice (1) is linear. Direct calculations prove that either the second lattice is linear as well, or we arrive to a particular case of the pair $\left(X_{8}, Y_{8}\right)$.

\section{Associated equations}

\subsection{Hyperbolic PDE systems}

In accordance to the nondegeneracy conditions the equations (1), (2) can be solved with respect to the variables $u_{ \pm 1}, v_{ \pm 1}$ :

$$
\begin{array}{lrl}
u_{1}=\tilde{F}\left(u_{x}, u, v\right), & v_{-1}=\tilde{G}\left(u, v, v_{x}\right), \\
u_{-1}=\tilde{P}\left(u_{y}, u, v\right), & v_{1}=\tilde{Q}\left(u, v, v_{y}\right) .
\end{array}
$$

Therefore, these variables can be eliminated from the expressions for the mixed derivatives and some system of partial differential equations appears. The original lattices define its Bäcklund auto-transformation. The form of the system is given by equations

$$
u_{x y}=f_{4} u_{x} u_{y}+f_{3} u_{x}+f_{2} u_{y}+f_{1}, \quad v_{x y}=g_{4} v_{x} v_{y}+g_{3} v_{x}+g_{2} v_{y}+g_{1},
$$

where the coefficients depend on $u, v$. Indeed, consider the equalities

$$
u_{x y}=F_{u_{1}} T(P)+F_{u} u_{y}+F_{v} v_{y}=P_{u_{-1}} T^{-1}(F)+P_{u} u_{x}+P_{v} v_{x} .
$$

In the first one the variables $u_{1}, v_{1}$ should be eliminated only and we see that the expression for $u_{x y}$ does not depend on $v_{x}$ and is linear in $u_{y}$. Analogously, elimination of $u_{-1}, v_{-1}$ in the second equality proves that the expression for $u_{x y}$ does not depend on $v_{y}$ and is linear in $u_{x}$. The formula for $v_{x y}$ is proved analogously.

Proposition 6. In virtue of the lattices from the list 7.1, the variables $u, v$ satisfy the respective systems from the list 7.2 .

\subsection{Ruijsenaars-Toda lattices}

As we have already explained in the Introduction, elimination of the variable $v$ allows to rewrite the lattice (1) in the form of Ruijsenaars-Toda type lattice (4), and its symmetry (2) in the form (5). Moreover, the equation (6) is fulfilled as well. The triples of the lattices corresponding to the list 7.1 are enumerated in the list 7.3. The remarkable property is that elimination of $u$ instead of $v$ brings to the same equations. This can be proved without calculations since it is easy to see that the lattices from the list 7.1 are invariant with respect to the involution

$$
u_{n} \leftrightarrow \sigma v_{-n}, \quad x \rightarrow-x, \quad y \rightarrow-y,
$$


and the lattices from the list 7.3 are invariant with respect to the involution

$$
u_{n} \leftrightarrow \sigma u_{-n}, \quad x \rightarrow-x, \quad y \rightarrow-y,
$$

where $\sigma=1$ for $\left(X_{1}\right),\left(Y_{1}\right),\left(R_{1}\right)$ and $\sigma=-1$ in other cases. It should be noted that this property is not invariant under the general substitutions (7). For an arbitrary choice of the variables $u$ and $v$ the corresponding Ruijsenaars-Toda lattices coincide after a suitable point substitution. As to the lattices $\left(X_{7}\right),\left(Y_{7}\right),\left(X_{8}\right),\left(Y_{8}\right)$, they possess the above symmetry only if $\varepsilon=1$.

\subsection{Discrete Toda lattices}

Coincidence of the lattices (4) for the variables $u$ and $v$ allows to expand the equations on the square grid and leads to the discrete Toda lattices. More precisely, this property means that the equations (1), (2) define the Bäcklund auto-transformations for the lattices (4), (6) correspondingly. This allows to introduce the variable $u(n, m, x, y)$, so that the pair $(u(n), v(n))$ is identified with $(u(n, m), u(n, m+1))$ at arbitrary $m$. Let us rewrite the lattices from the list 7.1 denoting the shifts by the double subscripts:

$$
\begin{aligned}
& u_{x}=F\left(u_{0,1}, u, u_{1,0}\right)=G\left(u_{-1,0}, u, u_{0,-1}\right), \\
& u_{y}=P\left(u_{0,-1}, u, u_{1,0}\right)=Q\left(u_{-1,0}, u, u_{0,1}\right) .
\end{aligned}
$$

Then the following properties are valid:

1) equations $F=G$ and $P=Q$ are equivalent and can be rewritten as the discrete Toda lattice

$$
f\left(u_{0,1}, u\right)+\tilde{f}\left(u_{0,-1}, u\right)=g\left(u_{1,0}, u\right)+\tilde{g}\left(u_{-1,0}, u\right) ;
$$

2 ) equation (41) is consistent with the dynamics on $x$ and $y$, that is, the equations obtained by differentiating of this equation in virtue of (39) or (40) are its consequences;

3 ) the variables $u(n)=u(m, n)$ for any $m$ satisfy the Ruijsenaars-Toda lattices (4), (6);

4) the variables $u(m)=u(m, n)$ for any $n$ satisfy the analogous lattices (possibly, with the different right hand sides);

5) the variables along the diagonals $u(n)=u(m-n, n)$ satisfy a Toda type lattice with respect to the $x$-derivatives

$$
u_{x x}=\tilde{A}\left(u_{x}, u_{1}, u, u_{-1}\right),
$$

and the variables along the complementary diagonals $u(n)=u(m+n, n)$ satisfy a Toda type lattice with respect to the $y$-derivatives

$$
u_{y y}=\tilde{C}\left(u_{y}, u_{1}, u, u_{-1}\right) .
$$

6) all these equations are Lagrangian.

\section{$5 \quad$ Non-Hamiltonian lattices}

The pairs $\left(X_{7}, Y_{7}\right)$ and $\left(X_{8}, Y_{8}\right)$ are of interest as counter-examples to the statement that existence of one symmetry implies the existence of the whole hierarchy. These lattices do not possess the higher symmetries at arbitrary $f, g$ and $\varepsilon$. In particular, the necessary integrability conditions fail for the associated lattices of the form (4). In accordance to [13] the simplest of these conditions reads

$$
D_{x}\left(\log A_{u_{1, x}}\right) \in \operatorname{Im}(T-1) .
$$


The direct computation proves that elimination of $v$ from equations $\left(X_{7}\right)$ brings to the lattice

$$
u_{x x}=\frac{\left(u_{x}+1\right)\left(\varepsilon u_{x}-1\right)}{1+\varepsilon}\left(\frac{u_{1, x}+1}{u_{1}+\varepsilon u}-\frac{\varepsilon u_{-1, x}-1}{u+\varepsilon u_{-1}}\right)
$$

and the above condition is fulfilled for this lattice only if $\varepsilon=1$.

It turns out, however, that the nature of these counter-examples is not too profound. It is easy to see that the non-invertible substitution $U=v-u_{1}, V=\varepsilon u-v_{1}$ reduces the lattices $\left(X_{8}, Y_{8}\right)$ to the disjoint pairs

$$
U_{x}=\varepsilon f\left(U_{-1}\right)-f\left(U_{1}\right), \quad U_{y}=0 ; \quad V_{x}=0, \quad V_{y}=\varepsilon p\left(V_{-1}\right)-p\left(V_{1}\right) .
$$

Analogous, but not so evident substitution exists for the pair $\left(X_{7}, Y_{7}\right)$ as well: the variables

$$
U=\frac{(1+\varepsilon)(u+v)}{\left(\varepsilon v_{-1}-u\right)\left(\varepsilon v-u_{1}\right)}, \quad V=\frac{(1+\varepsilon)(u+v)}{\left(\varepsilon u_{-1}-v\right)\left(\varepsilon u-v_{1}\right)}
$$

satisfy the equations

$$
\begin{aligned}
& U_{x}=U\left(U_{1}+U-\varepsilon\left(U+U_{-1}\right)\right), \quad U_{y}=0 ; \\
& V_{x}=0, \quad V_{y}=-V\left(V_{1}+V-\varepsilon\left(V+V_{-1}\right)\right) .
\end{aligned}
$$

In both cases the quantities $U(n)$ are the local first integrals for one lattice in the pair, and $V(n)$ for another one. In these variables the consistency of $x$ - and $y$-flows become trivial and is not related to integrability (although at $\varepsilon=1$ and for some $f, p$ equations may "accidentally" coincide with integrable Volterra type lattices [23]).

The fact that the local first integrals satisfy the closed lattice with respect to the second independent variable can be easily explained in the framework of the associated hyperbolic system. For the pair $\left(X_{7}, Y_{7}\right)$ it is of the form

$$
u_{x y}=\frac{\left(u_{x}+1\right)\left(u_{y}+1\right)}{u+v}, \quad v_{x y}=\frac{\left(v_{x}-1\right)\left(v_{y}-1\right)}{u+v}
$$

(notice that the units may be dropped, due to the substitution $u \rightarrow u-x-y, v \rightarrow v+$ $x+y$, however this leads to nonautonomous lattices). This is the Liouville equation type system [20, 21, 22]. Elimination of the shifts in the first integrals (42) leads to the invariants of this system, that is, to the quantities $I\left(u, v, u_{x}, v_{x}, \ldots\right), J\left(u, v, u_{y}, v_{y}, \ldots\right)$ which satisfy the properties $I_{y}=0, J_{x}=0$ in virtue of (44). It can be proved that, in the case of the hyperbolic system of the second order, all solutions of the equation $I_{y}=0$ are expressed through the $x$ derivatives of at most two basic invariants. Therefore, the invariants $U_{1}, U, U_{-1}$ must be related by some differential constraint, as the first equation (5) shows.

Finally, notice that the system (44) implies

$$
\left(\log u_{x y}\right)_{x y}=0, \quad\left(\log v_{x y}\right)_{x y}=0 .
$$

This gives the formula for the general solution

$$
u=a(x) b(y)+c(x)+d(y), \quad v=-u+\frac{\left(u_{x}+1\right)\left(u_{y}+1\right)}{u_{x y}}
$$

under assumption $a^{\prime} b^{\prime} \neq 0$, and this ansatz reduces the lattices $\left(X_{7}, Y_{7}\right)$ to the recurrent relations

$$
a_{1}=\frac{\varepsilon c^{\prime}-1}{a^{\prime}}, \quad c_{1}=a a_{1}-\varepsilon c ; \quad b_{1}=\frac{d^{\prime}+1}{b^{\prime}}, \quad d_{1}=\varepsilon\left(b b_{1}-d\right) .
$$

These relations are equivalent to some Volterra type lattice, for example the substitution $a \rightarrow \varepsilon^{n} a$ and scaling of $x$ brings to the equation (known to be integrable at $\varepsilon=1[23]$ )

$$
\varepsilon^{n} a_{n, x}=\frac{1}{a_{n+1}-a_{n-1}} .
$$




\section{Concluding remarks}

The integrable lattices of the form (1) or (4) admit the very important generalization: their right hand sides may contain parameters which depend on $n$ and define the discrete spectra added by the iterated Bäcklund transformations. Such generalizations are known, actually, for all lattices from the above lists, see e.g. [12, 7]. It turns out, however, that $n$-dependent lattices (1) are not consistent with the symmetries of the form (2), only with the higher symmetries of NLS type. The nature of this phenomena is not well understood for the present. It takes place in another situations as well, for example, the dressing chain $v_{n, x}+v_{n+1, x}=\left(v_{n}-v_{n+1}\right)^{2}+\alpha_{n}$ is consistent with equation $v_{n, y}=\left(v_{n+1}-v_{n-1}\right)^{-1}$ only if $\alpha_{n}=\alpha_{n+1}$ [24]. However, the exceptional pairs $\left(X_{7}, Y_{7}\right),\left(X_{8}, Y_{8}\right)$ may depend on $n$, for example, the following pair is consistent:

$$
\begin{array}{ll}
u_{n, x}=\frac{v_{n}+\left(1+\varepsilon_{n}\right) u_{n}+u_{n+1}}{\varepsilon_{n} v_{n}-u_{n+1}}, & v_{n, x}=\frac{v_{n-1}+\left(1+\varepsilon_{n-1}^{-1}\right) v_{n}+u_{n}}{v_{n-1}-\varepsilon_{n-1}^{-1} u_{n}}, \\
u_{n, y}=\frac{v_{n}+\left(1+\varepsilon_{n-1}^{-1}\right) u_{n}+u_{n-1}}{\varepsilon_{n-1}^{-1} v_{n}-u_{n-1}}, & v_{n, y}=\frac{v_{n+1}+\left(1+\varepsilon_{n}\right) v_{n}+u_{n}}{v_{n+1}-\varepsilon_{n} u_{n}} .
\end{array}
$$

Another sort of the nonautonomous generalizations is related to the master-symmetries of the lattices (1), (2), see [14, 25].

The multifield lattices also should be mentioned. Probably, the earliest example of such kind was found in $[26,27]$. It generalizes the pair $\left(X_{6}, Y_{6}\right)$ in the rational form:

$$
u_{x}=u_{1}+\langle u, v\rangle u, \quad-v_{x}=v_{-1}+\langle u, v\rangle v ; \quad u_{y}=\frac{u_{-1}}{\left\langle u_{-1}, v\right\rangle-1}, \quad-v_{y}=\frac{v_{1}}{\left\langle u, v_{1}\right\rangle-1} .
$$

The vectors $u, v$ satisfy, in virtue of these equations, the system

$$
u_{x y}=\langle u, v\rangle u_{y}+\left(\left\langle u_{y}, v\right\rangle-1\right) u, \quad v_{x y}=-\langle u, v\rangle v_{y}-\left(\left\langle u, v_{y}\right\rangle+1\right) v .
$$

However, an analog of the lattice $\left(R_{6}\right)$ is absent in this example.

\section{$7 \quad$ Lists}

\subsection{Consistent pairs of the Hamiltonian lattices}

$$
\begin{aligned}
& u_{x}=\frac{a}{v-u_{1}}-\frac{a_{v}}{2}, \quad v_{x}=\frac{a}{v_{-1}-u}+\frac{a_{u}}{2}, \\
& u_{y}=\frac{a}{u_{-1}-v}+\frac{a_{v}}{2}, \quad v_{y}=\frac{a}{u-v_{1}}-\frac{a_{u}}{2}, \\
& a=\alpha_{1} u^{2} v^{2}+\alpha_{2} u v(u+v)+\alpha_{3}\left(u^{2}+v^{2}\right)+\alpha_{4} u v+\alpha_{5}(u+v)+\alpha_{6} \text {, } \\
& H=\log \left(u_{1}-v\right)-\frac{1}{2} \log a, \quad R=-\log \left(u-v_{1}\right)+\frac{1}{2} \log a, \\
& u_{x}=\left(u-u_{1}\right)(u-v), \quad v_{x}=\left(v_{-1}-v\right)(u-v), \\
& u_{y}=\frac{u-u_{-1}}{v-u_{-1}}, \quad v_{y}=\frac{v_{1}-v}{v_{1}-u}, \\
& a=u-v, \quad H=u v-u_{1} v, \quad R=\log \left(u-v_{1}\right)-\log (u-v), \\
& u_{x}=\left(1+e^{u_{1}-u}\right)\left(1+e^{u-v}\right), \quad v_{x}=\left(1+e^{v-v_{-1}}\right)\left(1+e^{u-v}\right), \\
& u_{y}=\frac{1+e^{u_{-1}-u}}{1-e^{u_{-1}-v}}, \quad v_{y}=\frac{1+e^{v-v_{1}}}{1-e^{u-v_{1}}}, \\
& a=1+e^{v-u}, \quad H=-e^{u_{1}-v}-e^{u-v}, \quad R=\log \left(1-e^{v_{1}-u}\right)-\log \left(1+e^{v-u}\right),
\end{aligned}
$$




$$
\begin{aligned}
& u_{x}=e^{u_{1}-u}+e^{u_{1}-v}, \quad v_{x}=e^{v-v_{-1}}+e^{u-v_{-1}}, \\
& u_{y}=e^{u_{-1}-u}+e^{u-1}-v, \quad v_{y}=e^{v-v_{1}}+e^{u-v_{1}}, \\
& a=1+e^{v-u}, \quad H=-e^{u_{1}-v}, \quad R=-e^{u-v_{1}}, \\
& u_{x}=e^{u_{1}-v}+e^{u-v}, \quad v_{x}=e^{u-v_{-1}}+e^{u-v}, \\
& u_{y}=e^{v-u-1}+e^{v-u}, \quad v_{y}=e^{v_{1}-u}+e^{v-u}, \\
& a=1, \quad H=-e^{u_{1}-v}-e^{u-v}, \quad R=e^{v_{1}-u}+e^{v-u}, \\
& u_{x}=e^{u_{1}-u}+e^{u-v}, \quad v_{x}=e^{v-v_{-1}}+e^{u-v}, \\
& u_{y}=\frac{e^{u-1}-u}{e^{u-1}-v-1}, \quad v_{y}=\frac{e^{v-v_{1}}}{e^{u-v_{1}}-1}, \\
& a=-e^{v-u}, \quad H=e^{u_{1}-v}+\frac{1}{2} e^{2(u-v)}, \quad R=\log \left(1-e^{u-v_{1}}\right) .
\end{aligned}
$$

\subsection{Hyperbolic PDE systems}

$$
\begin{aligned}
& a u_{x y}=a_{u} u_{x} u_{y}+2 \tilde{a}\left(u_{x}-u_{y}\right)+\tilde{a} a_{v}-\tilde{a}_{v} a, \\
& a v_{x y}=a_{v} v_{x} v_{y}-2 \tilde{a}\left(v_{x}-v_{y}\right)+\tilde{a} a_{u}-\tilde{a}_{u} a, \\
& 4 \tilde{a}=a a_{u v}-a_{u} a_{v}
\end{aligned}
$$

$$
\begin{aligned}
u_{x y} & =\frac{\left(u_{y}-1\right) u_{x}}{u-v}+u_{y}(u-v), & v_{x y} & =-\frac{\left(v_{y}-1\right) v_{x}}{u-v}-v_{y}(u-v), \\
u_{x y} & =-\frac{u_{x}\left(u_{y}-1\right)}{1+e^{u-v}}+u_{y}\left(1+e^{u-v}\right), & v_{x y} & =\frac{v_{x}\left(v_{y}-1\right)}{1+e^{u-v}}-v_{y}\left(1+e^{u-v}\right), \\
u_{x y} & =-\frac{u_{x} u_{y}}{1+e^{u-v}}+1+e^{u-v}, & v_{x y} & =\frac{v_{x} v_{y}}{1+e^{u-v}}-1-e^{u-v}, \\
u_{x y} & =e^{u-v} u_{y}-e^{v-u} u_{x}, & v_{x y} & =e^{v-u} v_{x}-e^{u-v} v_{y}, \\
u_{x y} & =-u_{x} u_{y}+2 e^{u-v} u_{y}-1, & v_{x y} & =v_{x} v_{y}-2 e^{u-v} v_{y}+1 .
\end{aligned}
$$

\subsection{Ruijsenaars-Toda lattices}

$$
\begin{aligned}
& \left\{\begin{array}{l}
u_{x x}=\left(u_{x}^{2}+r(u)\right)\left(\frac{u_{1, x}}{T(a)}-\frac{u_{-1, x}}{a}+\frac{1}{2} \partial_{u} \log (T(a) a)\right)-\frac{1}{2} r^{\prime}(u), \\
u_{y y}=\left(u_{y}^{2}+r(u)\right)\left(\frac{u_{1, y}}{T(a)}-\frac{u_{-1, y}}{a}+\frac{1}{2} \partial_{u} \log (T(a) a)\right)-\frac{1}{2} r^{\prime}(u), \\
\left(u_{x} u_{y}-r(u)\right)\left(u_{1}-u_{-1}\right)+\left(u_{x}+u_{y}\right)\left(a+\frac{1}{2} a_{u_{-1}}\left(u_{1}-u_{-1}\right)\right)=0 \\
a=a\left(u, u_{-1}\right), \quad 4 r(u)=2 a a_{u_{-1} u_{-1}}-a_{u_{-1}}^{2},
\end{array}\right. \\
& \left\{\begin{array}{l}
u_{x x}=u_{x}\left(\frac{u_{1, x}}{u_{1}-u}-\frac{u_{-1, x}}{u-u_{-1}}-u_{1}+2 u-u_{-1}\right) \\
u_{y y}=-u_{y}\left(u_{y}-1\right)\left(\frac{u_{1, y}}{u_{1}-u}-\frac{u_{-1, y}}{u-u_{-1}}\right) \\
u_{x} u_{y}=\left(1-u_{y}\right)\left(u_{1}-u\right)\left(u-u_{-1}\right) \\
u_{x x}=u_{x}\left(\frac{u_{1, x}}{1+e^{u-u_{1}}}-\frac{u_{-1, x}}{1+e^{u_{-1}-u}}-e^{u_{1}-u}+e^{u-u_{-1}}\right) \\
u_{y y}=-u_{y}\left(u_{y}-1\right)\left(\frac{u_{1, y}}{1+e^{u-u_{1}}}-\frac{u_{-1, y}}{1+e^{u-1}-u}\right) \\
u_{x} u_{y}=\left(u_{y}-1\right)\left(e^{u_{1}-u}+1\right)\left(e^{u-u_{-1}}+1\right)
\end{array}\right.
\end{aligned}
$$




$$
\begin{aligned}
& \left\{\begin{array}{l}
u_{x x}=u_{x}\left(u_{1, x}-u_{-1, x}-e^{u_{1}-u}+e^{u-u_{-1}}\right) \\
u_{y y}=u_{y}\left(u_{-1, y}-u_{1, y}-e^{u_{-1}-u}+e^{u-u_{1}}\right) \\
u_{x}=u_{y} e^{u_{1}-u_{-1}}
\end{array}\right. \\
& \left\{\begin{array}{l}
u_{x x}=\frac{u_{1, x} u_{x}}{1+e^{u-u_{1}}}-\frac{u_{x} u_{-1, x}}{1+e^{u-1}-u}, \\
u_{y y}=\frac{u_{1, y} u_{y}}{1+e^{u-u_{1}}}-\frac{u_{y} u_{-1, y}}{1+e^{u-1}-u}, \\
u_{x} u_{y}=\left(e^{u_{1}-u}+1\right)\left(e^{u-u-1}+1\right),
\end{array}\right.
\end{aligned}
$$

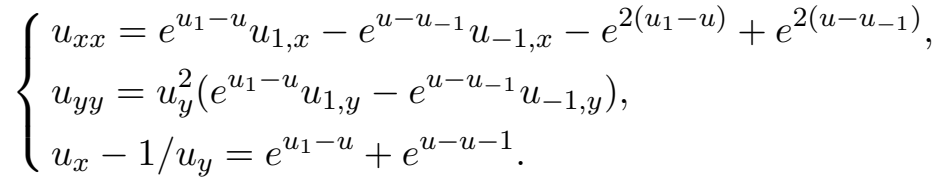

\section{Acknowledgements}

This research was supported by the RFBR grant \# 04-01-00403.

[1] Ruijsenaars S.N.M., Relativistic Toda system, Preprint Stichting Centre for Mathematics and Computer Sciences, Amsterdam, 1986.

[2] Ruijsenaars S.N.M., Relativistic Toda systems, Comm. Math. Phys., 1990, V.133, 217-247.

[3] Bruschi M., Ragnisco O., On a new integrable Hamiltonian system with nearest-neighbour interaction, Inverse Problems, 1989, V.5, 983-998.

[4] Suris Yu.B., Discrete time generalized Toda lattices: complete integrability and relation with relativistic Toda lattices, Phys. Lett. A, 1990, V.145, 113-119.

[5] Adler V.E., Shabat A.B., On the one class of the Toda chains, Theor. Math. Phys., 1997, V.111, 323-334.

[6] Adler V.E., Shabat A.B., Generalized Legendre transformations, Theor. Math. Phys., 1997, V.112, 935-948.

[7] Adler V.E., Shabat A.B., First integrals of the generalized Toda lattices, Theor. Math. Phys., 1998, V.115, 349-358.

[8] Marikhin V.G., Shabat A.B., Integrable lattices, Theor. Math. Phys., 1999, V.118, 217-228.

[9] Adler V.E., Marikhin V.G., Shabat A.B., Canonical Bäcklund transformations and Lagrangian chains, Theor. Math. Phys., 2001, V.129, 163-183.

[10] Ragnisco O., Santini P.M., A unified algebraic approach to integral and discrete evolution equations, Inverse Problems, 1990, V.6, 441-452.

[11] Shabat A.B., Yamilov R.I., Symmetries of nonlinear lattices, Leningrad Math. J., 1991, V.2, 377-400.

[12] Adler V.E., Yamilov R.I., Explicit auto-transformations of integrable chains, J. Phys. A: Math. Gen., 1994, V.27, 477-492.

[13] Yamilov R.I., Symmetry approach to the classification from the point of view of the differential-difference equations. Theory of transformations, Doctoral Thesis, Ufa, 2000.

[14] Adler V.E., Shabat A.B., Yamilov R.I., Symmetry approach to the integrability problem, Theor. Math. Phys., 2000, V.125, 1603-1661.

[15] Adler V.E., Discretizations of the Landau-Lifshitz equation, Theor. Math. Phys., 2000, V.124, 897-908.

[16] Hirota R., Nonlinear partial difference equations. II. Discrete-time Toda equation, J. Phys. Soc. Japan, 1977, V.43, 2074-2078.

[17] Suris Yu.B., Bi-Hamiltonian structure of the $q d$ algorithm and new discretizations of the Toda lattice, Phys. Lett. A, 1995, V.206, 153-161.

[18] Adler V.E., On the structure of the Bäcklund transformations for the relativistic lattices, J. Nonlinear Math. Phys., 2000, V.7, 34-56, nlin.SI/0001072.

[19] Adler V.E., Suris Yu.B., Q4: Integrable master equation related to an elliptic curve, Internat. Math. Res. Not., 2004, V.47, 2523-2553, nlin.SI/0309030.

[20] Zhiber A.V., Ibragimov N.H., Shabat A.B., Liouville type equations, DAN SSSR, 1979, V.249, 26-29.

[21] Sokolov V.V., Zhiber A.V., On the Darboux integrable hyperbolic equations, Phys. Lett. A, 1995, V.208, 303-308. 
[22] Zhiber A.V., Sokolov V.V., Exactly solvable hyperbolic equations of the Liouville type, Uspekhi Mat. Nauk, 2001, V.56, N 1, 63-106.

[23] Yamilov R.I., On classification of discrete evolution equations, Uspekhi Mat. Nauk, 1983, V.38, N 6, $155-156$.

[24] Adler V.E., Shabat A.B., Dressing chain for the acoustic spectral problem, Theor. Math. Phys., 2006, V.149, 1324-1337, nlin.SI/0604008.

[25] Nijhoff F., Hone A., Joshi N., On a Schwarzian PDE associated with the KdV hierarchy, Phys. Lett. A, 2000, V.267, 147-156, solv-int/9909026.

[26] Svinolupov S.I., Yamilov R.I., The multi-field Schrödinger lattices, Phys. Lett. A, 1991, V.160, 548-552.

[27] Merola I., Ragnisco O., Tu G.-Z., A novel hierarchy of integrable lattices, Inverse Problems, 1994, V.10, 1315-1334, solv-int/9401005. 